\title{
HAMARTOMA FIBROLIPOMATOSO E MACRODISTROFIA LIPOMATOSA: ANÁLISE DOS ACHADOS CLÍNICOS E DE IMAGEM EM QUATRO CASOS, COM REVISÃO DA LITERATURA*
}

\author{
Rafael Darahem de Souza Coelho ${ }^{1}$, Marcelo Novelino Simão ${ }^{2}$, Clóvis Simão Trad ${ }^{3}$
}

Resumo O hamartoma fibrolipomatoso neural e a macrodistrofia lipomatosa são doenças raras, com achados de imagem característicos. Radiografias simples, estudos de ressonância magnética e prontuários médicos de quatro pacientes foram revisados. Em um paciente, foi realizada cirurgia com confirmação histopatológica. Em três pacientes, os achados clínicos e de imagem foram considerados suficientes para o diagnóstico. Descrevemos dois casos de macrodistrofia lipomatosa isolada, um caso de hamartoma fibrolipomatoso do nervo mediano e um caso de coexistência das duas condições. As radiografias simples, nos casos de macrodistrofia lipomatosa, mostraram aumento difuso de partes moles e estruturas ósseas, com estrias radiotransparentes entremeadas às fibras musculares. Os dois casos de hamartoma fibrolipomatoso ocorreram no nervo mediano, com achados, à ressonância magnética, de fascículos nervosos espessados com baixo sinal nas imagens ponderadas em T1 e T2, com infiltração homogênea de gordura entre estes, aparecendo com alto sinal em T1 e baixo sinal em T2 com saturação de gordura. 0 aspecto do nervo aos cortes axiais é de "cabo coaxial". Nota-se extensão para a ramificação dos nervos, aspecto típico desta lesão. As características de imagem da macrodistrofia lipomatosa e do hamartoma fibrolipomatoso neural, principalmente pela ressonância magnética, permitiram o diagnóstico preciso destas condições freqüentemente coexistentes. Unitermos: Macrodistrofia lipomatosa. Hamartoma fibrolipomatoso. Ressonância magnética. Doença de nervos periféricos. Nervo mediano.

Abstract Fibrolipomatous hamartoma and macrodystrophia lipomatosa: imaging and clinical data analysis of four cases and review of the literature.

Fibrolipomatous hamartoma of nerve and macrodystrophia lipomatosa are rare conditions that present characteristic imaging findings. The plain films, magnetic resonance imaging studies and medical records of four patients were reviewed. The lesion was resected in one patient allowing histological confirmation of the diagnosis. Diagnosis was based on imaging and clinical findings in three patients who had unavailable biopsy data. We describe two cases of isolated macrodystrophia lipomatosa, one case of fibrolipomatous hamartoma and one case where both conditions were found. In the macrodystrophia lipomatosa cases plain films demonstrated enlargement of osseous and soft tissue structures in the affected limbs, with fatty streaks interspersed along the muscle fibers. The median nerve was affected in the cases of fibrolipomatous hamartoma. Magnetic resonance imaging findings included nerve fascicle thickening characterized by low signal on both T1 and T2-weighted sequences, and surrounding fat, which appears as high signal on T1 and low signal on T2-weighted sequences with fat saturation. Both nerves had a "coaxial cable" appearance on axial images. The fibrolipomatous hamartoma extended to the nerve branches, which is a typical finding. The typical imaging findings of macrodystrophia lipomatosa and fibrolipomatous hamartoma of nerve, particularly at magnetic resonance imaging examination, allowed a reliable diagnosis of these frequently coexistent conditions.

Key words: Macrodystrophia lipomatosa. Fibrolipomatous hamartoma. Magnetic resonance imaging. Peripheral nerve disease. Median nerve.

\footnotetext{
* Trabalho realizado no Hospital das Clínicas da Faculdade de Medicina de Ribeirão Preto da Universidade de São Paulo (FMRP-USP), Ribeirão Preto, SP.

1. Médico Residente em Radiologia do Hospital das Clínicas da FMRP-USP.

2. Médico Radiologista Assistente do Setor de Radiologia do Hospital das Clínicas da FMRP-USP.

3. Médico Radiologista Docente do Setor de Radiologia do Hospital das Clínicas da FMRP-USP.

Endereço para correspondência: Dr. Rafael Darahem de Souza Coelho. Rua Prudente de Morais, 577, apto. 12 Ribeirão Preto, SP, 14015-100. E-mail: rdarahem@terra. com.br

Recebido para publicação em 25/6/2002. Aceito, após revisão, em 16/7/2002.
}

\section{INTRODUÇÃO}

O hamartoma fibrolipomatoso neural é lesão benigna rara, descrita inicialmente em $1953^{(\mathbf{1})}$. O achado histológico característico é o espessamento de fascículos nervosos, entremeados por infiltração gordurosa e fibrose endo e perineural ${ }^{(2)}$. Acomete mais freqüentemente o nervo mediano, com relatos de envolvimento, também, dos nervos ulnar, radial, digitais, plantares, cranianos e isquiático ${ }^{(3-9)}$. Em $27 \%$ a $66 \%$ dos casos há associação com macrodactilia do membro envolvido, na forma de macrodistrofia lipomatosa $^{(2,10)}$. Esta é uma forma rara de gigantismo localizado, congênita e não-hereditária, descrita inicialmente por Feriz, em $1925^{(11)}$, caracterizada por crescimento de todos os elementos mesenquimais na distribuição de um nervo específico, com predominância do tecido fibroadiposo ${ }^{(12)}$. O envolvimento do 
nervo na macrodistrofia lipomatosa, quando presente, assume o mesmo aspecto do hamartoma fibrolipomatoso ${ }^{(\mathbf{1 3})}$.

\section{MATERIAIS E MÉTODOS}

Foi realizada busca no arquivo do Departamento de Radiologia de nossa instituição e feita a análise retrospectiva dos prontuários médicos e dos exames de imagem por dois médicos assistentes e um médico residente quanto às características clínicas (idade, sexo, sintomas) e de imagem (ressonância magnética - RM - em todos os quatro casos, radiografia simples em três casos). Em um dos pacientes foi realizada cirurgia, com confirmação histopatológica do diagnóstico. Em três pacientes, os achados clínicos e de imagem foram considerados suficientes para o diagnóstico. Os exames de RM foram realizados em aparelho Siemens Magnetom Vision ${ }^{\circledR}$ de 1,5 Tesla, seguindo protocolo da instituição, em cortes axiais, coronais e sagitais.

\section{RESULTADOS}

Foram identificados dois casos de macrodactilia (macrodistrofia lipomatosa) sem envolvimento aparente de estruturas neurais (pacientes 1 e 2), um caso de hamartoma fibrolipomatoso sem macrodactilia (paciente 4), e um caso de hamartoma fibrolipomatoso associado a macrodactilia (paciente 3 ). Os achados clínicos estão resumidos na Tabela 1 .

A idade dos pacientes, na apresentação, variou de 5 a 62 anos (idade média de 23,2 anos). Três dos quatro pacientes eram do sexo feminino. Os sintomas consistiam de dor (paciente 4) e limitação funcional e estética (demais pacientes).

As lesões neurais se localizavam no nervo mediano $(\mathrm{n}=2)$. Nos casos de ma- crodactilia, os dígitos envolvidos foram o primeiro quirodáctilo $(\mathrm{n}=2)$, o segundo quirodáctilo $(\mathrm{n}=1)$ e o segundo e terceiro pododáctilos $(\mathrm{n}=1)$. Nenhum dos pacientes tinha história familiar de macrodactilia ou neurofibromatose, e nenhum deles apresentava estigmas cutâneos.

Nos pacientes com macrodactilia (pacientes 1, 2 e 3 -Figuras 1, 2 e 3), as radio- grafias simples mostraram aumento difuso de partes moles nas regiões afetadas, em alguns casos (Figuras 1 e 3) identificando-se estrias radiotransparentes correspondentes às áreas de infiltração gordurosa. Observou-se, ainda, aumento do comprimento e largura de estruturas ósseas, que preservavam o trabeculado habitual. O paciente 1 apresentava elevações radiolucentes no

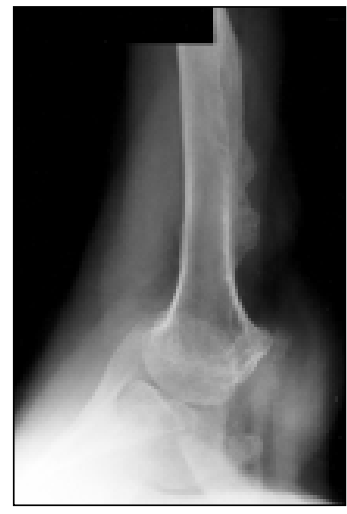

A

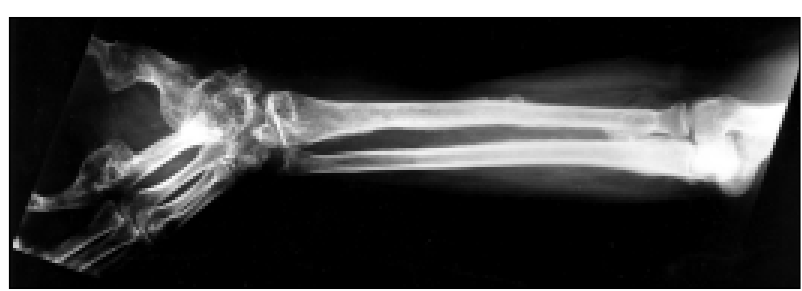

C

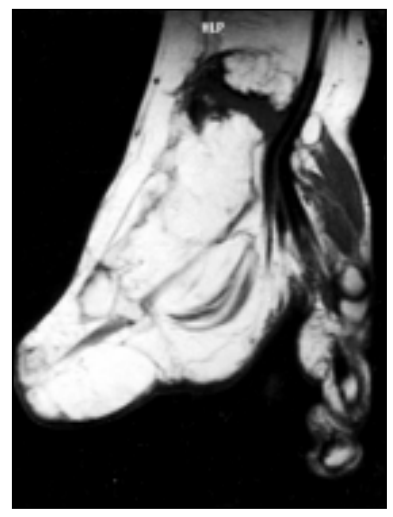

D

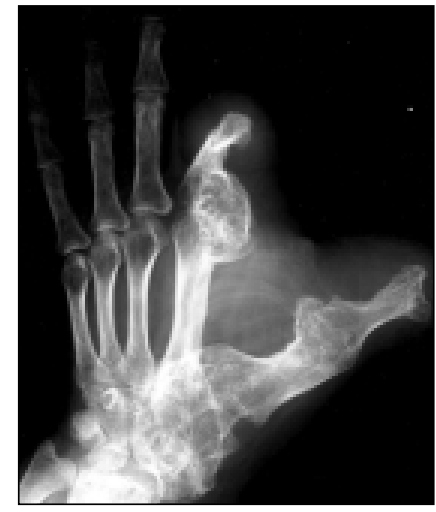

B

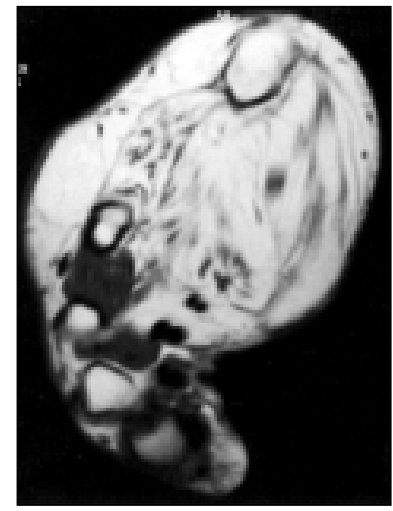

E
Figura 1. Caso 1. Em A, B C, radiografias simples mostram aumento difuso das partes moles, com estrias radiotransparentes referentes a gordura, e deformidade com alargamento ósseo em toda a extensão deste membro, de forma mais proeminente no primeiro e segundo quirodáctilos. Ausência cirúrgica da falange distal do segundo dedo. Em D, E e F, imagens de ressonância magnética ponderadas em T1 nos planos coronal (D) e axial (E,F) com alto sinal nas partes moles da região tenar, devido à infiltração gordurosa. Em F é demonstrado o nervo mediano, que tem aspecto presenvado.

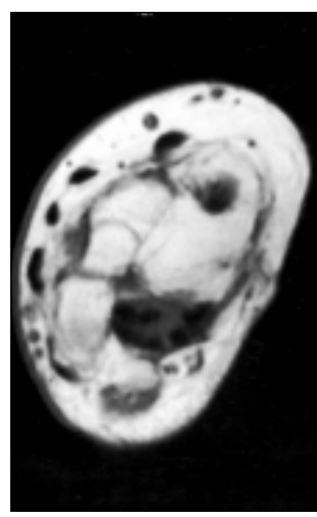

$\mathbf{F}$

Tabela 1 Dados clínicos.

\begin{tabular}{|c|c|c|l|l|l|l|}
\hline Paciente & Idade & Sexo & \multicolumn{1}{|c|}{ Macrodactilia } & Acometimento neural & \multicolumn{1}{|c|}{ Achados clínicos } \\
\hline 1 & 62 & Fem. & $\begin{array}{l}\text { Sim, primeiro e } \\
\text { segundo dedos da mão } \\
\text { Sim, segundo e terceiro } \\
\text { dedos do pé } \\
\text { Sim, polegar }\end{array}$ & Não & $\begin{array}{l}\text { Não } \\
\text { Sim, nervo mediano }\end{array}$ & $\begin{array}{l}\text { Aumento do antebraço e do primeiro e segundo } \\
\text { dedos da mão desde a infância } \\
\text { Aumento do segundo e terceiro dedos do pé } \\
\text { esquerdo desde o nascimento } \\
\text { Aumento de volume do antebraço e polegar } \\
\text { direitos desde o nascimento } \\
\text { Tumoração na face volar do punho esquerdo há } \\
\text { um ano, com dor }\end{array}$ \\
\hline
\end{tabular}




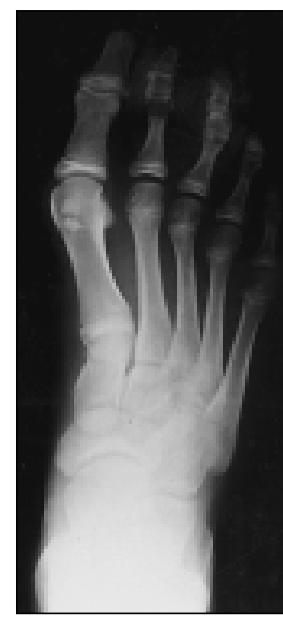

A

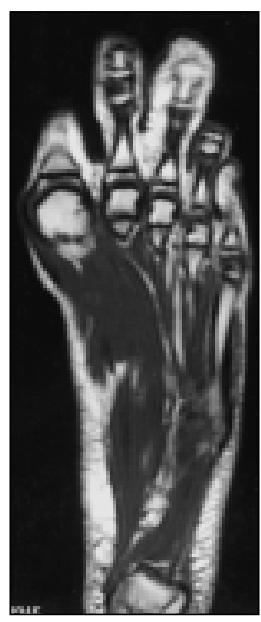

B

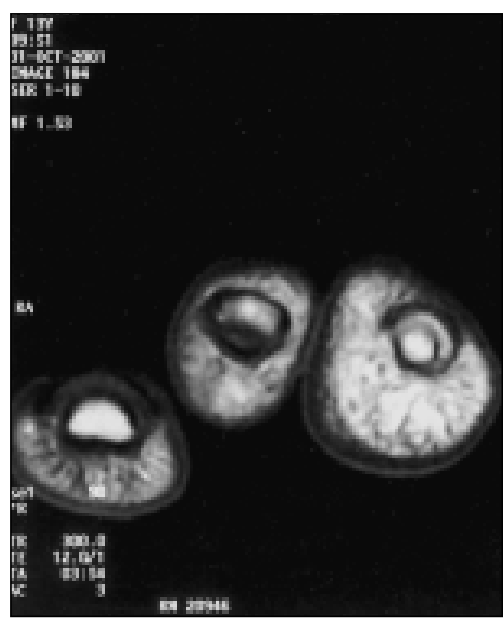

C

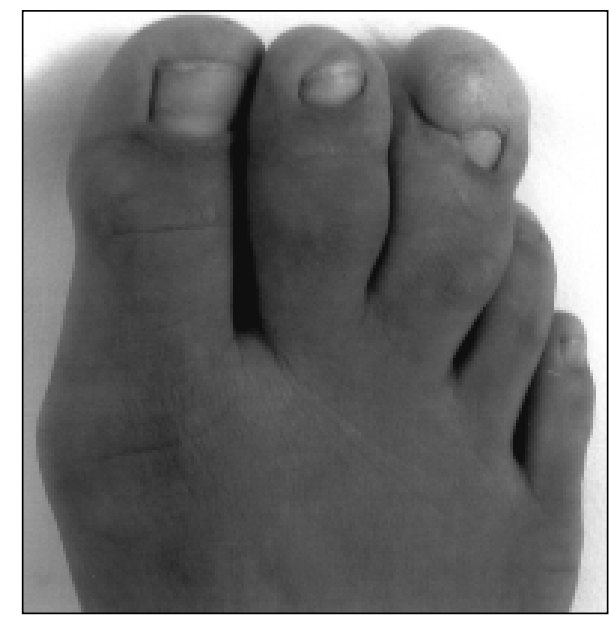

D

Figura 2. Caso 2. Macrodactilia do segundo e terceiro pododáctilos, demonstrada na radiografia simples em $\mathbf{A}$. Em $\mathbf{B}$ e $\mathbf{C}$, imagens de ressonância magnética ponderadas em T1 definem o aumento tanto das estruturas ósseas como das partes moles, com infiltração gordurosa difusa inferida pelo alto sinal. Em $\mathbf{D}$, correlação com a imagem do pé acometido.

Figura 3. Caso 3. Macrodistrofia lipomatosa associada a hamartoma fibrolipomatoso do nervo mediano. Em A observa-se aumento de volume ósseo e de partes moles no primeiro quirodáctilo/região tenar, aspecto também demonstrado em $\mathbf{B}$ pela ressonância magnética. Em C e D observase o nervo mediano no plano transverso adquirindo o aspecto de "cabo coaxial" (setas), com hipersinal em T1 entremeado a fascículos nervosos espessados com baixo sinal. Em E nota-se que este espessamento se estende para as ramificações do nervo mediano (pontas de setas).

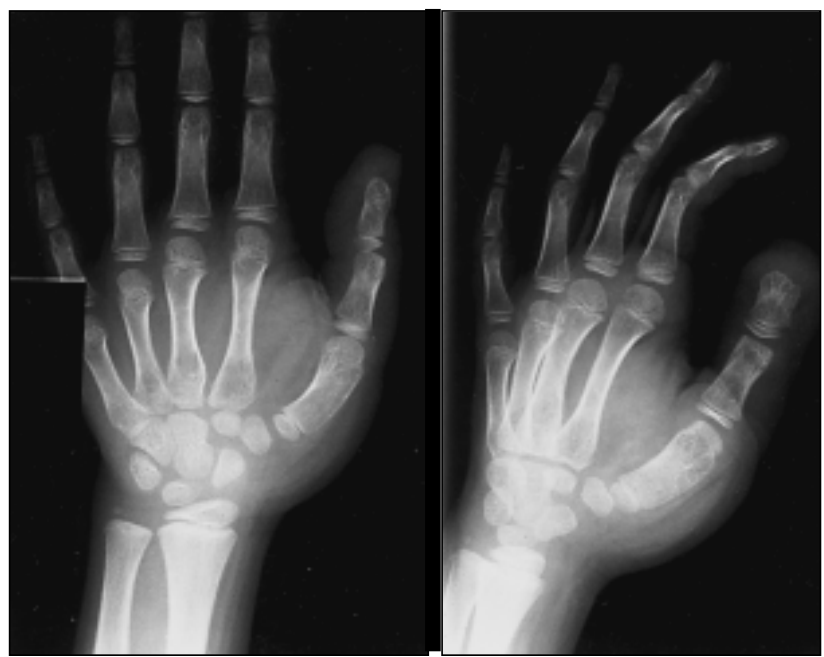

A

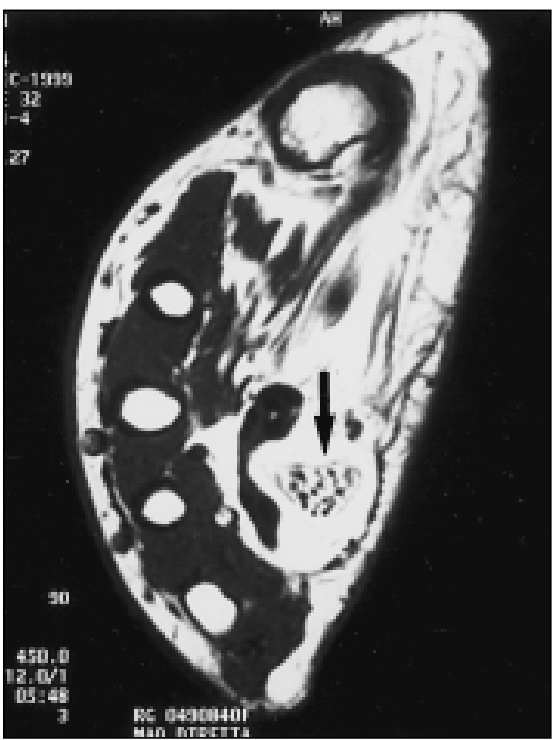

C

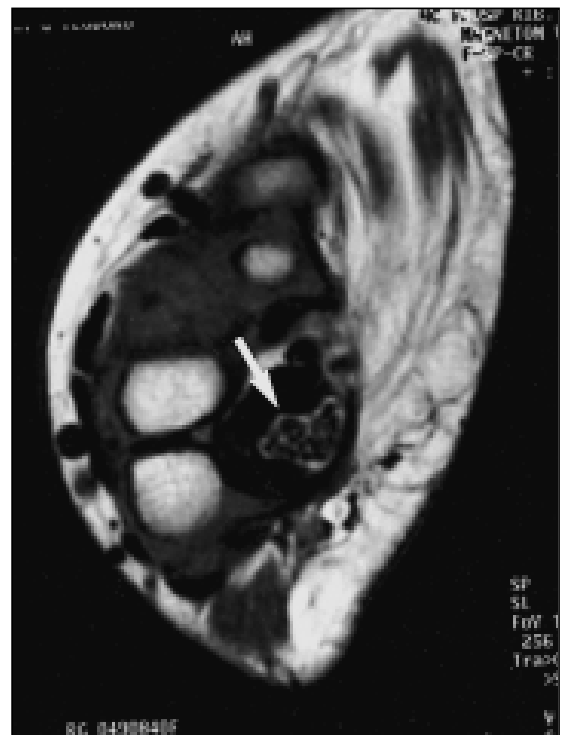

D

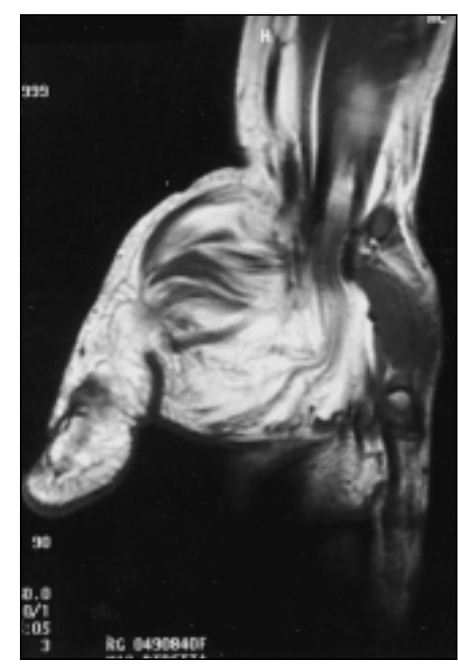

B

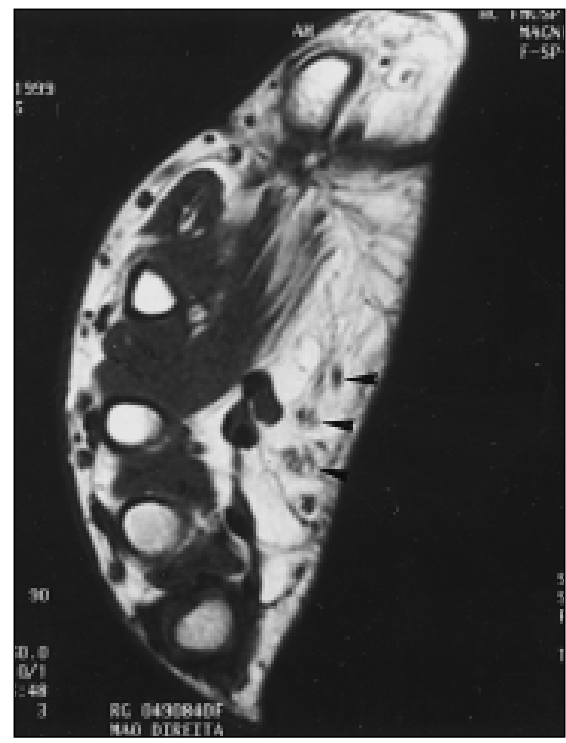

E 


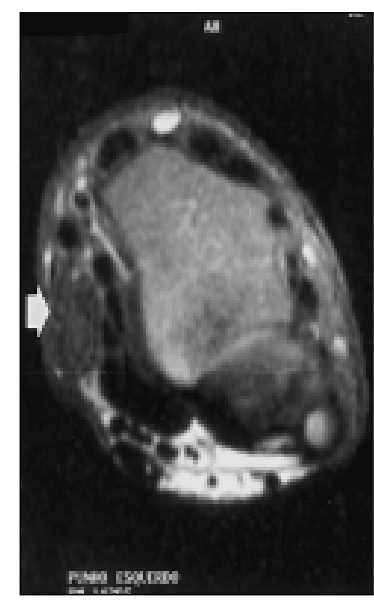

A

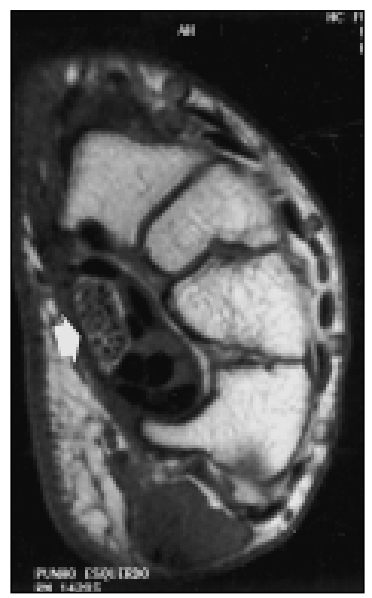

B

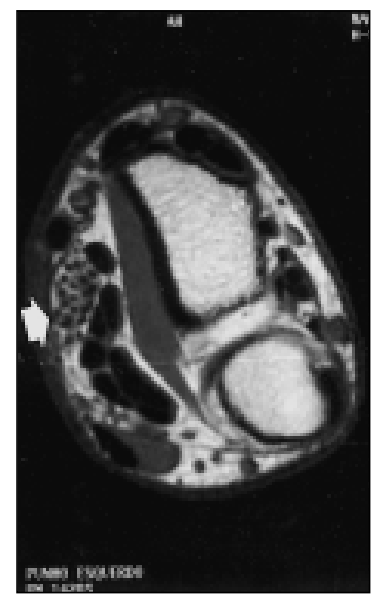

C

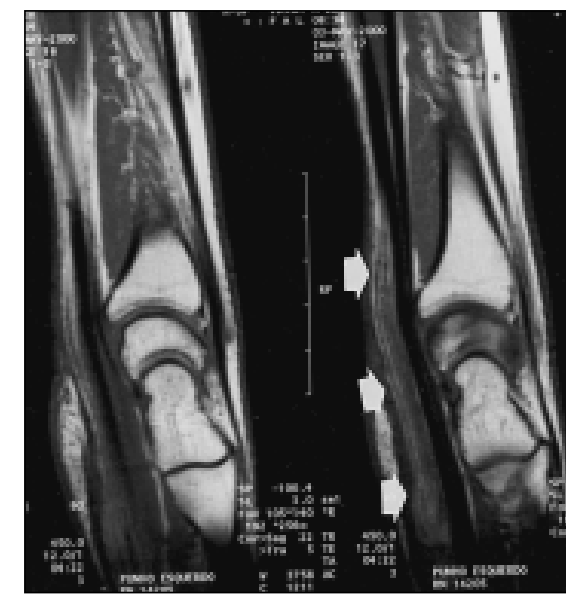

D

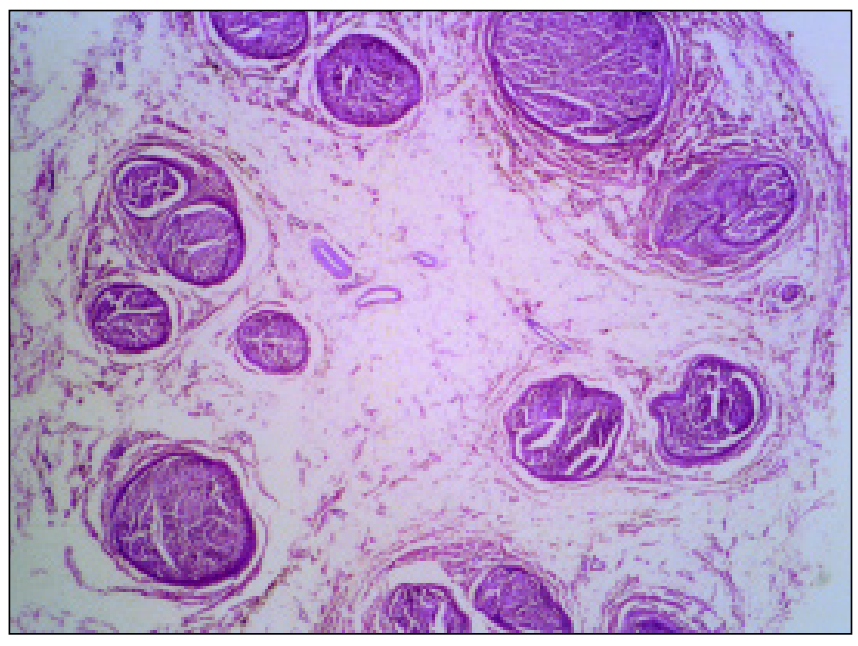

E

Figura 4. Caso 4. Em A, B e C, imagens de ressonância magnética no plano axial demonstram o nervo mediano espessado (setas), com baixo sinal dos fascículos nervosos em T1 e T2, hipersinal em T1 (B) e hipossinal em T2 (A) da gordura circundante. Em D, a imagem no plano sagital evidencia o nervo mediano (setas) em seu maior eixo, difusamente espessado, demonstrando claramente a grande extensão e pouca delimitação da lesão. Em $\mathbf{E}$, exame anatomopatológico em corte transversal em pequeno aumento com vários fascículos nervosos em meio a abundante infiltração gordurosa. Em $\mathbf{F}$, maior aumento individualizando fascículo com fibrose endo e perineural.

periósteo do rádio e úmero (Figuras $1 \mathrm{~A}$ e 1C), que podem corresponder a acúmulos de material adiposo, embora não haja comprovação histológica. As regiões mais distais e superfícies ventrais apresentavam envolvimento mais acentuado. Nestes pacientes, a RM (Figuras 1, 2 e 3) demonstrou áreas de infiltração gordurosa difusa entre as fibras musculares e tecido subcutâneo e hipertrofia das estruturas ósseas.

Nos pacientes 3 e 4, os exames de RM evidenciaram espessamento difuso do nervo mediano, com baixo sinal dos fascículos nervosos nas seqüências ponderadas em T1 e T2, além de aumento homogêneo do conteúdo gorduroso entre os fascículos, apresentando alto sinal em T1 e baixo sinal em T2 com supressão de gordura (Fi- guras 3 e 4). Aos cortes axiais, observouse o aspecto típico de "cabo coaxial" do hamartoma fibrolipomatoso. O exame histopatológico do nervo mediano ressecado do paciente 4 demonstrou a fibrose peri e epineural e infiltração gordurosa interfascicular (Figuras 4E e 4F), como demonstrado no corte transversal à RM.

\section{DISCUSSÃO}

O hamartoma fibrolipomatoso representa um crescimento anormal do tecido fibroadiposo da bainha nervosa. Também conhecido como hamartoma lipomatoso, lipofibroma e infiltração lipomatosa neural, acomete o nervo mediano, exclusivamente, em mais de $80 \%$ dos $\operatorname{casos}^{(2)}$. Esta maior freqüência no nervo mediano não tem causa bem definida, sendo uma possibilidade o fato de este nervo tornar-se sintomático mais precocemente que os demais, devido à sua compressão pelo retináculo flexor no túnel do carpo ${ }^{(9)}$. Ocorre com maior freqüência em crianças, sendo considerado, por alguns, um tumor congênito $^{(2)}$, e por outros, um hamartoma cujo crescimento é estimulado por irritação ou inflamação do nervo, infreqüentemente associado a trauma ${ }^{(\mathbf{1 4 - 1 6})}$. Os sinais e sintomas precoces são mínimos. Tardiamente, estão relacionados à compressão do nervo no túnel do carpo e incluem dor, déficits motores e parestesia.

Patologicamente, o tumor é uma massa amarelada de consistência borrachosa, 
apresentando fibrose no perineuro e epineuro e infiltração do tecido conjuntivo interfascicular por adipócitos maduros, à microscopia. Os fascículos nervosos geralmente têm aparência normal ${ }^{(\mathbf{1 2})}$. O tratamento inclui exploração, liberação do túnel do carpo e eventualmente biópsia e excisão cirúrgica, havendo controvérsia em relação aos resultados desta última ${ }^{(17)}$.

Nossos dois casos de hamartoma fibrolipomatoso demonstram os achados à RM descritos previamente ${ }^{(\mathbf{1 7 - 1 9 )}}$, incluindo o aspecto considerado patognomônico de "cabo coaxial"(9). Os fascículos nervosos aumentados estão distribuídos homogeneamente pela bainha nervosa, apresentam baixo sinal nas seqüências ponderadas em T1 e T2. A infiltração homogênea de gordura entre os fascículos nervosos aparece com alto sinal em imagens ponderadas em T1 e baixo sinal em imagens ponderadas em T2 com supressão de gordura.

O diagnóstico diferencial, do ponto de vista clínico, inclui o lipoma intraneural, que à RM aparece como uma massa focal bem delimitada e individualizada em relação aos fascículos nervosos, em oposição à infiltração difusa característica do hamartoma fibrolipomatoso. Outras lesões a serem consideradas são o neuroma traumático, o neurofibroma plexiforme e o hemangioma, todos eles apresentando alto sinal nas imagens ponderadas em T2 com supressão de gordura, o que não ocorre no hamartoma fibrolipomatoso ${ }^{(9)}$.

O hamartoma fibrolipomatoso pode estar associado à macrodistrofia lipomatosa. Na maior série descrita ${ }^{(2)}$, dos 26 casos de hamartoma fibrolipomatoso, sete associavam-se à macrodistrofia lipomatosa. A macrodistrofia lipomatosa é uma forma de gigantismo localizado de um ou mais dígitos, neste último caso invariavelmente adjacentes. Barsky ${ }^{(20)}$, em 1967, revisando 64 casos de macrodactilia, encontrou predominância de acometimento do segundo e terceiro dedos, correspondendo ao território de inervação do nervo mediano. Embora o gigantismo localizado seja geralmente detectado ao nascimento, a apresentação é usualmente mais tardia, por razões cosméticas ou quando alterações degenerativas secundárias causam limitação funcio- nal. O achado patológico marcante é o aumento no tecido fibroadiposo, envolvendo a medula óssea, periósteo, músculos, bainhas nervosas e tecido subcutâneo ${ }^{(\mathbf{1 2})}$. O envolvimento neural pode ser proeminente. Quando coexistem, a relação causal entre o hamartoma fibrolipomatoso e a macrodistrofia lipomatosa não é bem estabelecida. Nos casos de macrodistrofia lipomatosa sem envolvimento aparente do nervo, há casos descritos de surgimento de aumento tardiamente, sugerindo ser uma evolução da doença ${ }^{(13)}$.

Os achados de imagem à radiografia simples $^{(\mathbf{1 2 , 2 2})}$ são um aumento de volume das estruturas ósseas e partes moles, mais proeminente na extremidade distal e na face ventral do membro acometido, além de envolvimento periosteal, aspectos bem demonstrados no paciente 1 (Figura 1). Áreas radiotransparentes refletindo um aumento do tecido gorduroso são geralmente detectáveis nas partes moles ${ }^{(\mathbf{1 2})}$. As falanges mostram-se alongadas e alargadas. À RM, define-se o aumento difuso do conteúdo de gordura nas partes moles. O principal diagnóstico diferencial é a neurofibromatose. Do ponto de vista clínico, a neurofibromatose difere da macrodistrofia lipomatosa por ser hereditária, pela coexistência de manchas cutâneas café-com-leite, pelo fato de poder ser bilateral e com envolvimento de dígitos não-contíguos. Aos exames de imagem, a neurofibromatose não apresenta as radiolucências em partes moles e, à RM, os neurofibromas são detectados como áreas localizadas de aumento de sinal em imagens ponderadas em T2. Outros diagnósticos diferenciais de macrodactilia incluem a síndrome de KlippelTrenaunay-Weber, hemangiomatose e linfangiomatose.

Embora tenhamos comprovação histológica de somente um dos dois casos de hamartoma fibrolipomatoso, o outro apresentava características de imagem idênticas ao nosso caso biopsiado e aos demais casos descritos na literatura. Em todos os casos a RM demonstrou os aspectos típicos, permitindo o diagnóstico preciso do hamartoma fibrolipomatoso e a caracterização dos casos de macrodactilia como macrodistrofia lipomatosa.

\section{REFERÊNCIAS}

1. Mason ML. Proceedings of the American Society for Surgery of the Hand: presentation of cases. J Bone Joint Surg Am 1953;35:273-5.

2. Silverman TA, Enzinger FM. Fibrolipomatous hamartoma of nerve: a clinicopathologic analysis of 26 cases. Am J Surg Pathol 1985;9:7-14.

3. Gouldesbrough DR, Kinny SJ. Lipofibromatous hamartoma of the ulnar nerve at the elbow: brief report. J Bone Joint Surg Br 1989;71:331-2.

4. Jacob RA, Buchino JJ. Lipofibroma of the superficial branch of the radial nerve. J Hand Surg (Am) 1989;14:704-6.

5. Herrick RT, Godsil RD, Widener JH. Lipofibromatous hamartoma of the radial nerve: a case report. J Hand Surg (Am) 1980;5:211-3.

6. Hirakawa E, Miki H, Kobayashi S, Ohmori M, Arima N. Lipofibromatous hamartoma of nerve in the foot. Acta Pathol Jpn 1993;43:265-7.

7. Steentoft J, Sollerman C. Lipofibromatous hamartoma of a digital nerve. Acta Orthop Scand 1990; 61:181-2.

8. Berti E, Roncaroli F. Fibrolipomatous hamartoma of a cranial nerve. Histopathology 1994;24:3912.

9. Marom EM, Helms CA. Fibrolipomatous hamartoma: pathognomonic on MR imaging. Skeletal Radiol 1999;28:260-4.

10. Amadio PC, Reiman HM, Dobyns JH. Lipofibromatous hamartoma of nerve. J Hand Surg (Am) 1988;13:67-76.

11. Kelikian H. Macrodactily. In: Congenital deformities of the hand and forearm. Philadelphia, PA: Saunders, 1974;610-60.

12. Goldman AB, Kaye JJ. Macrodystrophia lipomatosa: radiographic diagnosis. AJR 1977;128:101-5.

13. Brodwater BK, Major NM, Goldner RD, Layfield LJ. Macrodystrophia lipomatosa with associated fibrolipomatous hamartoma of the median nerve. Pediatr Surg Int 2000;16:216-8.

14. Bibbo C, Warren AM. Fibrolipomatous hamartoma of nerve. J Foot Ankle Surg 1994;33:64-71.

15. Callison JR, Thoms OJ, White WL. Fibro-fatty proliferation of the median nerve. Plast Reconstr Surg 1968;42:403-13

16. Rowland SA. Lipofibroma of the median nerve in the palm. J Bone Joint Surg Am 1967;49:1309-13.

17. Cavallaro MC, Taylor JAM, Gorman JD, Haghighi $\mathrm{P}$, Resnick D. Imaging findings in a patient with fibrolipomatous hamartoma of the median nerve. AJR 1993;1161:837-8.

18. De Maeseneer M, Jaovisidha S, Lenchik L, et al. Fibrolipomatous hamartoma: MR imaging findings. Skeletal Radiol 1997;26:155-60.

19. Boren WL, Henry REC Jr, Wintch K. MR diagnosis of fibrolipomatous hamartoma of nerve: association with nerve territory-oriented macrodactyly (macrodystrophia lipomatosa). Skeletal Radiol 1995;24:296-7.

20. Barsky AJ. Macrodactyly. J Bone Joint Surg Am 1967;49:1255-65.

21. Blacksin M, Barnes FJ, Lyons MM. MR diagnosis of macrodystrophia lipomatosa. AJR 1992;158: 1295-7.

22. Gupta SK, Sharma OP, Sharma SV, Sood B, Gupta S. Macrodystrophia lipomatosa: radiographic observations. Br J Radiol 1992;65:769-73. 